\title{
Successful placement of a deflectable decapolar catheter via the right femoral vein approach in a patient with coronary sinus ostial atresia and persistent left superior vena cava
}

\author{
Choon Ta Ng, ${ }^{1}$ Jason See, ${ }^{2}$ Chee Wan Lee, ${ }^{3}$ Chi-Keong Ching ${ }^{1}$
}

'Department of Cardiology, National Heart Centre Singapore, Singapore ${ }^{2}$ Department of Cardiology, Changi General Hospital, Singapore

${ }^{3}$ Department of Cardiology, Khoo Teck Pat Hospital, Singapore

\section{Correspondence to} Dr Chi-Keong Ching, Cardiac Pacing and

Electrophysiology, Department of Cardiology, National Heart Centre Singapore, 5 Hospital Drive, 169609 Singapore; ching.chi.keong@nhcs.com.sg

Accepted 6 August 2014

\section{ABSTRACT}

The authors illustrate the successful ablation of a leftsided posterior accessory pathway via a retrograde aortic approach in a patient with coronary ostial atresia associated with persistent left-sided superior vena cava. This is an extremely rare anomaly which should be considered by cardiac electrophysiologists when there is difficulty cannulating the coronary sinus via the right atrial route. Awareness of this route obviates the need for additional venous access.

\section{CASE REPORT}

A 22-year-old man with no past medical history presented with frequent palpitations. A 12-lead resting ECG showed supraventricular tachycardia that correlated with his symptoms. Transthoracic echocardiogram revealed normal ejection fraction with no structural abnormality.

He underwent an invasive cardiac electrophysiology (EP) study. Via the right femoral vein, the authors were unable to cannulate the coronary sinus (CS) with a deflectable decapolar catheter. Multiple attempts at locating the CS os with contrast injection through a Swartz Guiding sheath (SL2, St Jude Medical) were also unsuccessful. The possibility of CS ostial atresia which is usually accompanied by a persistent left superior vena cava (PLSVC) was considered. In this patient, the
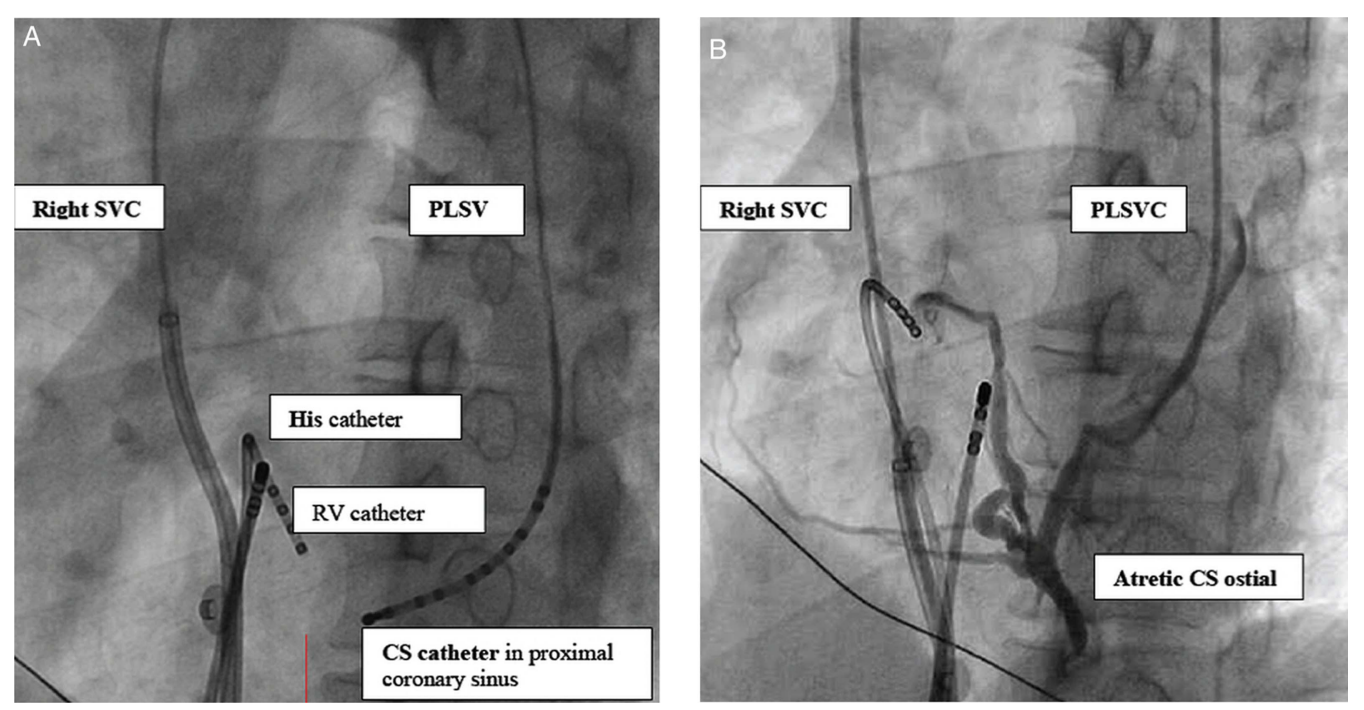

Figure 1 (A) (left): Left anterior oblique (LAO) $45^{\circ}$ view: Successful placement of a deflectable decapolar catheter within the coronary sinus (CS) by advancing from the right atrium to the right superior vena cava (SVC) across the innominate vein into the persistent left SVC (PLSVC) in the patient. (B) (right): LAO $45^{\circ}$ view: Venogram demonstrating venous drainage via the PLSVC, and the atresia of CS ostium. RV, right ventricle.

authors managed to advance the deflectable decapolar catheter from the right atrium to the rightsided SVC, across the innominate vein, inferiorly to the left-sided SVC and successfully position it within the CS with its distal electrode at the proximal CS (figure 1A).

EP study was performed with induction of orthodromic reciprocating tachycardia involving a concealed left-sided lateral accessory pathway. This was successfully ablated via a retrograde aortic approach, aided by the CS catheter electrograms. Post ablation, a selective venogram (figure 1B) confirmed the presence of a PLSVC with CS ostial atresia.

CS ostial atresia associated with PLSVC is an extremely rare anomaly ${ }^{1-3}$ which should be considered by cardiac electrophysiologists when there is difficulty cannulating the CS via the right atrial route despite multiple attempts and with various long sheaths. The presence of retrograde flow from the CS to the left superior vena cava, and via the innominate vein to the right superior vena cava ${ }^{1}$ allows us to successfully place a decapolar catheter within the CS. This step is crucial in performing an EP study and guiding ablation of left-sided accessory pathways. Awareness of the possibility of this route obviates the need to obtain additional venous access via the left subclavian or the internal jugular veins.

To cite: $\mathrm{Ng} \mathrm{CT}$, See $\mathrm{J}$, 2014;6:128-129.

doi:10.1136/heartasia-2014010563 
Contributors CTN: involved in the conceptualisation and drafting of the manuscript. CWL: involved in the conceptualisation, drafting and review of the manuscript. JS: involved in the review of the manuscript. C-KC: involved in the conceptualisation and review of the manuscript.

Competing interests None.

Patient consent Not obtained.

Provenance and peer review Not commissioned; internally peer reviewed.

\section{REFERENCES}

1 Santoscoy R, Walters HL, Ross RD, et al. Coronary sinus ostial atresia with persistent left superior vena cava. Ann Thorac Surg 1996;61:879-82.

2 Yoshida S, Yokoe $\mathrm{H}$, Murakawa $\mathrm{K}$, et al. Radiofrequency catheter ablation of posterior paraseptal accessory pathway with atresia of the coronary sinus ostium. PACE 2012;35:e80-3.

$3 \mathrm{Kim} \mathrm{J}$, Kim JH, Chun KJ, et al. Left-sided accessory pathway with ostial atresia of the coronary sinus. PACE 2008;31:129-30. 